$\underset{\text { publications }}{\mathbf{C}} \mathbf{G}$

Rec. Nat. Prod. 16:3 (2022) 253-258

records of natural

products

\title{
Phenolic Acid Analogues from Balanophora laxiflora Inhibit Proliferation of In Vitro Acute Myeloid Leukemia Cells
}

\author{
Trinh Thi Thuy $\oplus^{1,2 * *}$, Ba Thi Cham $\oplus^{1,2}$, Nguyen Thi Thuy Linh $\oplus^{1,2}$, \\ Phan Thi Ngoc Bich $\oplus^{1,2}$, Sabrina Adorisio $\oplus^{3^{*}}$ \\ and Domenico V. Delfino $\oplus^{3,4 *}$
}

\begin{abstract}
${ }^{1}$ Department of Chemistry, Graduate University of Science and Technology, Vietnam Academy of Science and Technology, 18 Hoang Quoc Viet, Nghia Do, Cau Giay, Hanoi, Viet Nam ${ }^{2}$ Department of Natural Products Research, Institute of Chemistry, Vietnam Academy of Science and Technology, 18 Hoang Quoc Viet, Nghia Do, Cau Giay, Hanoi, Viet Nam ${ }^{3}$ Foligno Nursing School, Department of Medicine and Surgery, University of Perugia, Perugia, Italy ${ }^{4}$ Section of Pharmacology, Department of Medicine and Surgery, University of Perugia, Piazzale Severi, S. Andreadelle Fratte, 06132 Perugia, Italy
\end{abstract}

(Received June 28, 2021; Revised August 2, 2021; Accepted August 6, 2021)

\begin{abstract}
Four phenolic analogues isolated from Balanophora laxiflora; methyl gallate (1, MG), 4-hydroxy-3methoxycinnamaldehyde (2), 3-methoxycinnamic acid (3), and methyl caffeate (4, MC) were shown to have antiproliferative activity on acute myeloid leukemia (OCI-AML3) cells due to impaired cell cycle progression. Three of these compounds $(\mathbf{1}, \mathbf{3}$, and $\mathbf{4})$ caused increased apoptosis and decreased proliferationin cells. Interestingly, MC (compound 4) significantly decreased the percentage of cells in S phase and G2/M phase, and significantly increased apoptosis in cells at low concentrations. Treatment with these compounds increased Fas ligand production and caspase-8/3, but not caspase-9-dependent, apoptosis. These results suggest that dosing with these compounds decreases proliferation and increases apoptosis in cell lines derived from hematological cancers.
\end{abstract}

Keywords: Balanophora laxiflora Hemsl. ex Forbes \& Hemsl.; phenolic acid analogues; antiproliferative activity; caspase-8; acute myeloid leukemia cell (OCI-AML); human cancer cell lines. (C) 2021 ACG Publications. All rights reserved.

\section{Plant Source}

In the course of phytochemical studies of medicinal plants from Viet Nam, we investigate Balanophora laxiflora [1-5].

\footnotetext{
* Corresponding authors: Email: domenico.delfino@unipg.it; Phone: +39 75585 8328/+39 755858115 (D.V. Delfino) thuy@ich.vast.vn (T.T. Thuy); Phone: +84 978987562.
} 
Phenolic acid analogues inhibit acute myeloid leukemia cells

Balanophora laxiflora Hemsl. ex Forbes \& Hemsl. was collected at Na Hang district, Tuyen Quang province, Viet Nam in December, 2018. The species was identified by botanist Dr. Do Huu Thu, Institute of Ecology and Biological Resources, Vietnam Academy of Science and Technology, Hanoi, Vietnam (VAST). A voucher specimen is deposited at the Herbarium of Institute of Ecology and Biological Resources, VAST, Hanoi, Viet Nam.

\section{Previous Studies}

Balanophora is a genus belonging to the family Balanophoraceae, which contains approximately 120 species worldwide. Balanophora laxiflora Hemsl. ex Forbes \& Hemsl. (B. laxiflora) is a parasitic herb growing in Taiwan and northern Vietnam. B. laxiflora has been used in Vietnamese folk medicine as a tonic with antipyretic and antidotal properties as a treatment for hemorrhoids, stomach ache, hemoptysis, and to improve sexual performance [1-3]. Previous phytochemical studies of $B$. laxiflora led to the isolation of phenolic compounds and lignin [1-5]. Lignan compounds have been proven to possess DPPH radical and scavenging properties with antiinflammatory activities [6-8]. Phenolic compounds, especially caffeic acid analogues, have been reported to have antinociceptive, anticancer, antiproliferative, proapoptotic and anti-angiogenesis activities [9-14]. Previous studies have shown that treatment with MC (compound 4) has shown potential apoptotic activity on MCF-7 cells [11] and human breast cancer cells [12]. Recently, our group has extensively studied the effect of compounds isolated from a Vietnamese B. laxiflora. As part of our study, we identified four phenolic compounds (1-4) from this plant, namely methyl gallate (1, MG), 4-hydroxy-3-methoxycinnamaldehyde (2), 3-methxoxycinnamic acid (3), and methyl caffeate (4) [1]. Natural phenolic compounds have potential health benefits, and these caffeic acid derivatives have been predicted to act as chemopreventive agents and emerging adjuvants for cancer chemotherapy [15-16].

\section{Present Study}

In the present study, compounds 1-4 were isolated from aerial part of $B$. laxiflora by a modified method previously described [1], and detail described in the supplementary material. The structures of compounds 1-4 (Figure 1) were identified as methyl gallate (1), 4-hydroxy-3methoxycinnamaldehyde (2), 3-methxoxycinnamic acid (3), methyl caffeate (4) by extensive spectroscopic analysis including 1D and 2D NMR and HR-ESIMS experiments as well as by comparison with reported data [1] (see supporting information for spectral data). The presence of compounds 1 and $\mathbf{4}$ in the ethanolic extract from the same plant material proves that they are also natural constituents. The purity of MC exceeded $96 \%$ as determined by HPLC, and its chromatogram is shown in the supporting information (Figure S1). However, little is known about the antiproliferative activity of these compounds in acute myeloid leukemia. This study characterizes the antiproliferative, apoptotic, and cell cycle arrest effects of the phenolic compounds on acute myeloid leukemia cells (OCI-ALM3).

We characterized the effect of compounds 1-4 on the growth of OCI-AML3 cells by evaluating cell counts, apoptosis, and cell cycle progression. Among the four compounds, compound $\mathbf{4}$ was the most powerful and it was analyzed in depth. Compounds $\mathbf{1}$ and $\mathbf{3}$ at 150 and $300 \mu \mathrm{g} / \mathrm{mL}$ (Figure S6A) and 4 at 15.62 and $31.25 \mu \mathrm{g} / \mathrm{mL}$ (Figure 2A) significantly decreased the number of OCI cells, respectively, whereas compound 2 (that was toxic at higher concentrations) did not demonstrate any activity (Figure S6A). The decrease in cell numbers could be due to increased apoptosis, decreased proliferation, or both. Apoptosis of OCI-AML3 cells was examined using propidium iodide flow cytometry analysis, gating out necrotic cells by size (forward light scatter) [18-20]. Results show that treatment with compounds $\mathbf{1}$ and $\mathbf{3}$ significantly increased the occurrence of apoptosis at a concentration of 150 and $300 \mu \mathrm{g} / \mathrm{mL}$, respectively, (Figure S6B), whereas compound 4 was the most powerful since it significantly increased apoptosis at $15.62 \mu \mathrm{g} / \mathrm{mL}$ (Fig. 2B and C). Compound 2 was ineffective (Figure S6B). Thus, the decrease in OCI cell numbers was caused at least in part by increased cellular apoptosis.

In the cell cycle analysis, compounds 1, 2, 3 (Figure S7) and 4 (Figure. 3A and B) 
significantly increased the percentage of cells in $\mathrm{G}_{0} / \mathrm{G}_{1}$ phase, although compound $\mathbf{3}$ did it only at a $300 \mu \mathrm{g} / \mathrm{mL}$. Compounds 1, 2, 3 (at $300 \mu \mathrm{g} / \mathrm{mL}$ ) (Figure S7) and 4 (Figure. 3A and B) decreased the percentage of cells in S phase, whereas only compounds 3 (at $300 \mu \mathrm{g} / \mathrm{mL}$ ) (Figure S7) and $\mathbf{4}$ (Figure. $3 \mathrm{~A}$ and B) were able to decrease the percentage of cells in G2/M phase. We hypothesize that all compounds $(\mathbf{1}, \mathbf{2}, \mathbf{3}$, and $\mathbf{4})$ impaired the cell cycle by a similar mechanism [21]. Since MC (4) had the lowest MEC, it was chosen to further investigate its role in the caspase cascade, the hallmark of apoptotic cell death. Caspase activity was measured before and after treatment with compound $\mathbf{4}$ by western blot analysis. Cells treated with compound $\mathbf{4}$ activated caspase- 3 and caspase-8, but not caspase-9 (Figure 4A). Thus, compound 4 promoted OCI cell apoptosis, as demonstrated by the activation of caspase-3 via the caspase-8-dependent extrinsic pathway.<smiles>COC(=O)/C=C/c1ccc(O)c(O)c1</smiles>

Figure 1. Structures of isolated compounds 1 - 4 from Balanophora laxiflora

One way to trigger the extrinsic apoptotic pathway is to stimulate receptors of the TNF receptor family by their ligands. In order to see if this was the case, OCI cells with or without the addition of compound $\mathbf{4}$ were cultured and after 24 hours were harvested, their RNA extracted and a real-time has been performed to evaluate the expression of Fas ligand (FasL), TNF $\alpha$ (both belonging to the TNF family and causing apoptosis by extrinsic pathway) and TGF- $\beta$ that can promote apoptosis due to its function as a tumor suppressor and with the involvement of the activation of SMAD proteins and of AP1 transcription factor [22]. Figure 4B shows that compound 4 significantly increased the expression of FasL but not TNF- $\alpha$ and TGF- $\beta$. Thus, the increased expression of FasL confirmed that the extrinsic apoptotic pathway was triggered by compound 4.

Among these, compounds 1, 3 and $\mathbf{4}$ decreased cell number in OCI-AML3 cells. This study verified that methyl esters (compounds 1 and 4) displayed higher anti-leukemic effects than acidic esters (compound $\mathbf{3}$ ), while aldehyde (compound $\mathbf{2}$ ) had no effect on cells number. MC (compound 4) had a significant effect on cells at a much lower concentration than MG (compound 1), and exerted its effect by blocking cells at G0/G1 phase of the cell cycle although its effect in increasing apoptosis seemed to be the main mechanism since it doubled at a concentration as low as $15.62 \mu \mathrm{g} / \mathrm{mL}$. Apoptosis is the target of different drugs, such as glucocorticoids, used to treat lymphohematopoietic cancer [23] and, in particular, induction of apoptosis is achieved by different drugs to treat acute myeloid leukemia, a malignancy of hematopoietic system that remain a challenge despite the advancement in cancer biology and therapy [24]. In AML, drug targets were located in both the intrinsic (mediated by caspase-9) and extrinsic (mediated by caspase-8) apoptotic pathways. In the first pathway, Bcl-2 and Mcl-1 were targeted with good results, whereas results obtained by targeting the extrinsic pathway were less impressive, although good results were obtained by targeting XIAP and cIAP molecules [24]. In our work, compound $\mathbf{4}$ induced apoptosis by significantly increasing another extrinsic apoptotic pathway molecule, Fas ligand, able to induce caspase-8-dependent apoptosis by an autocrine mechanism. Thus, FasL can be added to the extrinsic apoptotic drug targets in AML cells and compound $\mathbf{4}$ from B. laxiflora could be useful in triggering its production, thus promoting AML apoptosis. Further in vivo studies will clarify if this can be a novel possible way to treat AML. 
Phenolic acid analogues inhibit acute myeloid leukemia cells

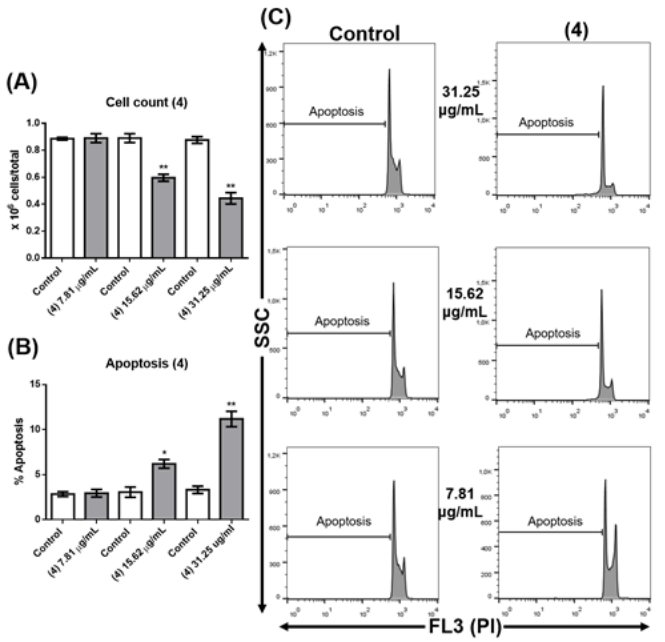

Figure 2. (A) Bars represent the number of viable cells counted after $24 \mathrm{~h}$ of treatment with vehicle (Control, white bars), or compound 4 at the concentrations reported on the $\mathrm{x}$-axis (Gray bars). (B) Effects of $\mathbf{4}$ on induction of apoptosis in OCI-AML3 cells. Bars represent the percentage of cell death after $24 \mathrm{~h}$ of treatment, with control vehicle (Control, white bars) or $\mathbf{4}$ at the concentrations reported on the $\mathrm{x}$-axis (gray bars). (C) Histograms from a representative experiment, in which propidium iodide (PI) staining is shown on the $\mathrm{x}$-axes on the logarithmic scale (FL3). Data from five independent experiments are reported as standard error of the mean (SEM). $* * p<0.01$,

$* \mathrm{p}<0.05$.

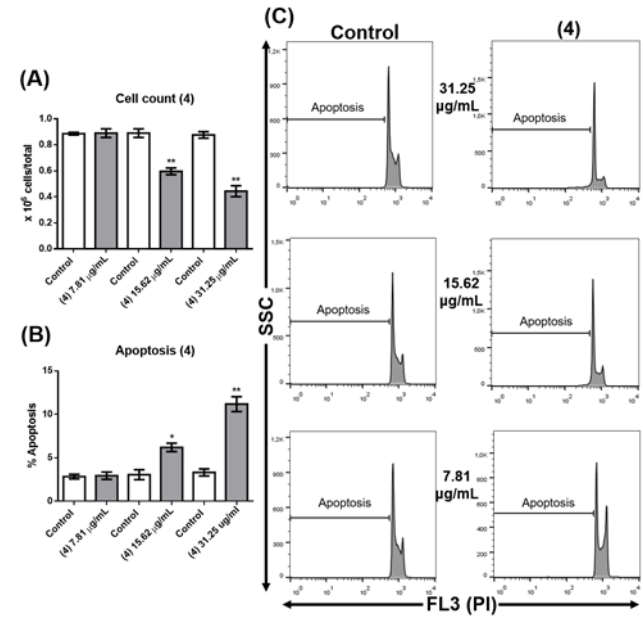

Figure 3. Effects of 4 on OCI-AML3 cell cycle progression. (A) Bars represent the percentage of cells in $\mathrm{G} 0 / \mathrm{G} 1, \mathrm{~S}$, or G2/M phase after $24 \mathrm{~h}$ of treatment with control DMSO vehicle (Control, white bars) or compound 4 at the concentrations reported in the $\mathrm{x}$-axes (Gray bars). (B) Panels show flow cytometry analyses of a representative experiment. Data from five independent experiments are reported as SEM. $* * \mathrm{p}<$ 0.01 .
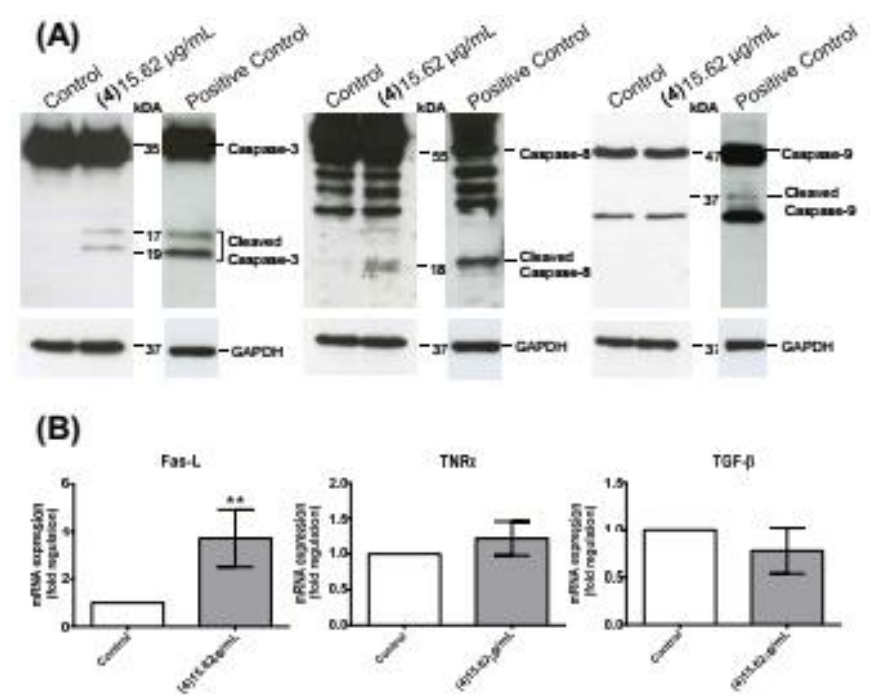

Figure 4. Effects of (4) on apoptotic pathways. (A) Western blot analysis of pro-caspase-3 (caspase3 ), activated caspase-3 (cleaved caspase 3), pro-caspase- 8 (caspase-8), activated caspase- 8 (cleaved caspase 8), pro-caspase-9 (caspase 9), and activated caspase-9 (cleaved caspase 9) in OCI-AML3 cells treated with vehicle (Control) or (4) at $15.62 \mu \mathrm{g} / \mathrm{mL}$ for $24 \mathrm{~h}$. Western blots are representative of five independent experiments. (B) Real-time PCR analyses from five independent experiments (mean \pm SEM) of Fas-L, TNF- $\alpha$, TGF- $\beta$, in cells treated with vehicle (Control) or $15.62 \mu \mathrm{g} / \mathrm{mL}(4), * * \mathrm{p}<0.01$. 
In conclusion, three compounds $(\mathbf{1}, \mathbf{3}$, and $\mathbf{4})$ decreased cell number in OCI-AML3 cells. This study verified that methyl esters (compounds 1 and 4) displayed higher antiproliferative effects than acidic esters (compound 3), while aldehyde (compound 2) had no effect on cells number. MC (compound 4) had a significant effect on cells at a much lower concentration than MG (compound 1). Hopefully, additional bioactive studies will clarify this effect and examine its potential therapeutic use.

\section{Acknowledgments}

This work was supported by the Vietnam Academy of Science and Technology (under grant number VAST.04.08/20-21).

\section{Conflict of interest statement}

We declare that we have no conflict of interest.

\section{Supporting Information}

Supporting information accompanies this paper on http://www.acgpubs.org/journal/records-ofnatural-products

\section{ORCID}

Trinh Thi Thuy: 0000-0001-5568-6925

Ba Thi Cham: 0000-0001-6690-8845

Nguyen Thi Thuy Linh: 0000-0003-2226-6064

Phan Thi Ngoc Bich: 0000-0002-1020-4010

Sabrina Adorisio: 0000-0001-9310-3487

Domenico Delfino: 0000-0003-1792-9468

\section{References}

[1] T. D. Dai, N. Q. Tien, N. N. Tuan, N. Q. An, T. T. T. Nga, T. T. Thuy and D. N. Quang (2017). Chemical constituents of Balanophora laxiflora Hemsley collected in Tuyen Quang. Part I. Chemical constituents of the less polar extracts, Vietnam J. Chem. 55(1), 48-51.

[2] T. D. Dai, T. T. Thuy, N. N. Tuan, N. Q. An, T. T. T. Nga, H. D. Cuong and N. Q. Tien (2018). Chemical constituents of Balanophoralaxiflora Hemsley collected in Tuyen Quang. Part III. Phenolic compounds, Vietnam J. Chem. 56(2), 254-259.

[3] B.H. Tai, N. X. Nhiem, P. H. Yen, T. H. Quang, N. T. Cuc, D. T. Trang, V.V. Doan, D. T. T. Hang, C. V. Minh and P.V. Kiem (2020). Three new muurolane-type sesquiterpene glycosides from the whole plants of Balanophora fungosa subsp. indica, Nat. Prod. Res. 34(20), 2964-2970

[4] T. D. Dai, N. Q. Tien, N. Q. An, T. T. T. Nga, T. T. Thuy and D. N. Quang (2016). Chemical constituents of Balanophora laxiflora Hemsley collected in Tuyen Quang. Part II. Glycosides compounds, Vietnam J. Chem. 54(6e2), 48-52.

[5] D. N. Quang, T. C. So, N. T. P. Thanh, L. T. P. Hoa, P. H. Dien, T. M. Luong, N. Q. Tung, L. D. Long, T. D. Dai and N. Q. Tien (2018). Balanochalcone, a new chalcone from Balanophora laxiflora Hemsl, Nat. Prod. Res. 2(7), 767-772.

[6] S. Kyung-Min, K. In-Tae, P. Young-Mi, H. Joohun, C. Jong-Won, P. Hee-Juhn, S. L. Yong and L. Kyung-Tae (2004). Anti-inflammatory effect of caffeic acid methyl ester and its mode of action through the inhibition of prostaglandin E2, nitric oxide and tumor necrosis factor- $\alpha$ production, Biochem. Pharmacol. 68, 2327-2336.

[7] W. F .Chiou, C. C. Shen and L. C. Lin (2011). Anti-inflammatory principles from Balanophora laxiflora, J. F. D. A. 19(4), 502-508.

[8] G. M. She, Y. J. Zhang and C. R. Yang (2009). Phenolic constituents from Balanophora laxiflora with DPPH radical-scavenging activity, Chem. Biodivers. 6, 875-880. 
Phenolic acid analogues inhibit acute myeloid leukemia cells

[9] C. B. Fa'tima, L. F. Caroline, A. Graziele, F. Mauricio, C. F. Valdir, A. Y. Rosendo and R. Niero (2009). Antinociceptive properties of caffeic acid derivatives in mice, Eur. J. Med. Chem. 44, 45964602.

[10] S. M. Fiuza, C. Gomes, L. J. Teixeira, M. T. Girao da Cruz, M. N. D. S. Cordeiro, N. Milhazes, F. Borgesa and M. P. M. Marquesa (2004). Phenolic acid derivatives with potential anticancer properties a structure-activity relationship study. Part 1: Methyl, propyl and octyl esters of caffeic and gallic acids, Bioor. Med. Chem. 12, 581-589.

[11] C. Balachandran, N. Emia, Y. Arun, Y. Yamamoto, B. Ahilan, B. Sangeetha, V. Duraipandiyan, I. Yoko, O. Akinao, S.Ignacimuthu, N.A. Al-Dhabi and P.T. Perumal. (2015). In vitro anticancer activity of methyl caffeate isolated from Solanum torvum Swartz. Fruit, Chem. Bio. Interact. 242, 81-90.

[12] F. Bailly, R. A. Toillon, O. Tomavo, N. Jouy, H. Hondermarck and P. Cotelle (2013). Antiproliferative and apoptotic effects of the oxidative dimerization product of methyl caffeate on human breast cancer cells, Bioorg Med. Chem. Lett. 23, 574-578.

[13] R. G. Gopalsamy, I. Savarimuthu, G. P. Michael and S. Ponnusamy (2011). Antihyperglycemic activity and antidiabetic effect of methyl caffeate isolated from Solanum torvum Swartz. fruit in streptozotocin induced diabetic rats, Eur. J. Pharmacol. 670, 623-631.

[14] W. Chieh-HsiKuo,Yueh-Hsiung and P.Chun-Hsu (2015). Caffeic acid derivatives for anti-angiogenesis Patent, US2016355493.

[15] S. P. Lee, G. Jun, E. Yoon, S. Park and C. Yang (2001). Inhibitory effect of methyl caffeate on Fos-JunDNA complex formation and suppression of cancer cell growth, Bull. Korean Chem. Soc. 22, 11311135 .

[16] N. J. Kang, K.W. Lee, B. J. Shin, S. K. Jung, M. K. Hwang and A. M. Bode (2009). Caffeic acid, a phenolic phytochemical in coffee, directly inhibits Fyn kinase activity and UVB-induced COX-2 expression, Carcinogenesis 30(2), 321-330.

[17] N. Pozzesi, S. Pierangeli, C. Vacca, L. Falchi, V. Pettorossi, M. P. Martelli, T. T. Thuy, P. T Ninh., A. M. Liberati, C. Riccardi, T. V. Sung and D.V. Delfino (2011). Maesopsin 4-O-beta-D-glucoside, a natural compound isolated from the leaves of Artocarpus tonkinensis, inhibits proliferation and upregulates HMOX1, SRXN1 and BCAS3 in acute myeloid leukemia, J. Chemother. 23(3), 150-157.

[18] C. Riccardi and I .Nicoletti (2006). Analysis of apoptosis by propidium iodide staining and flow cytometry, Nat. Protoc. 1(3), 1458-1461.

[19] B. T. Cham, N. T. T. Linh, D. T. Thao, N. T. H. Anh, N. T. Tam, B. K. Anh, I. Muscari, S. Adorisio, Sung TV, Thuy TT and D. Delfino (2020). Cell growth inhibition of saponin XII from Dipsacus japonicas Miq. on acute myeloid leukemia cells, Molecules 25, 3325.

[20] S. Adorisio, A .Fierabracci, I. Muscari, A. M. Liberati, L. Cannarile, T. T. Thuy, T. V. Sung, H. Sohrab, C. M. Hasan, E. Ayroldi and D. V. Delfino (2019). Fusarubin and anhydrofusarubin isolated from a Cladosporium species inhibit cell growth in human cancer cell lines, Toxins (Basel) 11(9), 503.

[21] D. V. Delfino, N. Pozzesi, S. Pierangeli, E. Ayroldi and A. Fierabracci A (2011). Manipulating thymic apoptosis for future therapy of autoimmune diseases, Curr. Pharm. Des. 17, 3108-3119.

[22] N. Shuster and K. Krieglstein (2002). Mechanisms of TGF-beta-mediated apoptosis, Cell Tissue Res. 307(1), 1-14.

[23] E. Ayroldi, L. Cannarile L, D.V .Delfino and C. Riccardi (2018). A dual role for glucocorticoid-induced leucine zipper in glucocorticoid function: tumor growth promotion or suppression?, Cell Death Dis. 9 , 463-475.

[24] J. H. Choi, J. M. Bogenberger and R. Tibes (2020). Targeting apoptosis in acute myeloid leukemia: Current status and future directions of Bcl-2 inhibition with Venetoclax and beyond, Target. Oncol. 15,147-162.

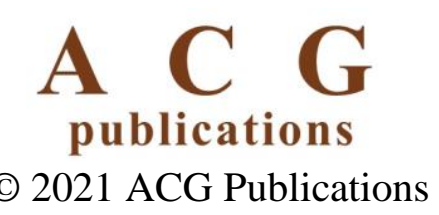

